\title{
Eventos estressores na família e indicativos de problemas de saúde mental em crianças com idade escolar
}

\author{
Stressor events in the family environment that are indicative \\ of mental health problems in children of school age
}

Mariana Bonati de Matos ${ }^{1}$ Ana Catarina Nova Cruz ${ }^{2}$ Samuel de Carvalho Dumith ${ }^{3}$ Natália da Costa Dias ${ }^{1}$ Renata Bonati Peters Carret ${ }^{1}$ Luciana de Avila Quevedo ${ }^{1}$

${ }^{1}$ Programa de PósGraduação em Saúde e Comportamento, Universidade Católica de Pelotas (UCPel). R. Gonçalves Chaves 373/411, Centro. 96015560 Pelotas RS Brasil. marianabonatidematos@ gmail.com ${ }^{2}$ Núcleo de Neurodesenvolvimento, UFPEL.

${ }^{3}$ Programa de PósGraduação em Epidemiologia, Departamento de Medicina Social, UFPEL.
Abstract The scope of this article is to evaluate the relationship between stressor events that occurred last year in the family of children and adolescents that are indicative of mental health problems in a sample of students from two schools in a city in southern Brazil. It involved a cross-sectional study with 1,075 students enrolled in two public elementary schools. The Strengths and Difficulties Questionnaire was used to assess emotional and behavioral factors of the child and the Social Readjustment Rating Scale (SRRS) of Holmes and Rahe (1967) to assess stressor events. The chisquare and Poisson regression test with robust variance adjustment for expressing the results in the prevalence ratio $(P R)$ and confidence intervals of $95 \%$ were used. The chances of presenting problems of hyperactivity were 1.42 (95\% CI 1.10 to 1.83) times higher in the intermediate tercile and 1.37 (95\% CI 1.06-1.78) in the higher tercile compared with the lower tercile. With respect to relationship problems the chances were of 1.49 (95\% CI 1.15 to 1.93) times higher in the higher tercile when compared with the lower tercile. The results suggest that environmental factors may be strongly related to the etiology of mental disorders in childhood and adolescence.

Key words Childhood, Adolescence, Stressor events, Mental health problems
Resumo O objetivo deste artigo é avaliar a relação entre eventos estressores ocorridos no último ano na família de crianças e adolescentes com indicativos de problemas de saúde mental em uma amostra de estudantes de duas escolas de uma cidade no sul do Brasil. Estudo transversal com 1.075 estudantes matriculados em duas escolas públicas de ensino fundamental (uma estadual e outra municipal). Foi utilizado o Strengths and Difficulties Questionnaire para avaliação de fatores emocionais e comportamentais da criança, e a Escala de Avaliação de Reajustamento Social de Holmes e Rahe (1967) para avaliar os eventos estressores. Foram utilizados o teste qui-quadrado e a regressão de Poisson, com ajuste robusto para variância, expressando os resultados em razão de prevalências $(R P)$ e intervalos de confiança de $95 \%$. As chances de apresentar problemas de hiperatividade foram 1,42 (IC 95\% 1,10-1,83) vezes maiores no tercil intermediário e 1,37 (IC 95\% 1,06-1,78) no tercil superior, quando comparados ao tercil inferior. Quanto aos problemas de relacionamento, as chances foram de 1,49 (IC $95 \%$ 1,15-1,93) vezes maiores no tercil superior ao serem comparados com o tercil inferior. Os resultados sugerem que fatores ambientais podem ser fortemente relacionados à etiologia dos transtornos mentais na infância e adolescência.

Palavras-chave Infância, Adolescência, Eventos estressores, Problemas de saúde mental 


\section{Introdução}

Os períodos da infância e da adolescência são considerados vulneráveis por serem fases do desenvolvimento nas quais o indivíduo encontra-se exposto a inúmeros fatores que contribuem para o surgimento de problemas físicos e de saúde mental ${ }^{1}$, podendo ser precursores de transtornos psiquiátricos e sociais ao longo da vida ${ }^{2}$.

Os problemas de saúde mental que ocorrem com maior frequência na infância e adolescência são os transtornos de conduta, de atenção e hiperatividade e emocionais, acometendo principalmente crianças e adolescentes em idade escolar, porém dados sobre prevalência são imprecisos devido à dificuldade de mensurá- $\operatorname{los}^{2-4}$. A avaliação da saúde mental neste período carece de instrumentos padronizados. O Strengths and Difficulties Questionnaire (SDQ), um dos poucos instrumentos utilizados, avalia as dificuldades da criança/adolescente através dos domínios emocional, conduta, hiperatividade e relacionamento com os pares.

De acordo com a literatura, problemas na saúde mental infantil podem estar relacionados tanto a fatores genéticos (pais com desordens afetivas, esquizofrenia, desordens antissociais, hiperatividade, déficit de atenção e isolamento), como biológicos (prematuridade, desnutrição, baixo peso, lesões cerebrais, atraso no desenvolvimento), os quais vêm sendo bastante estudados nos últimos 10 anos, sendo considerados fatores de risco ao desenvolvimento da criança ${ }^{6}$.

Apesar da influência genética e biológica, os fatores considerados psicossociais, como a família desestruturada, o desemprego, a pobreza e a dificuldade de acesso à saúde e educação, e os fatores ambientais, como doença na família, morte de alguém querido e até uma situação de divórcio, atualmente têm despertado a atenção dos pesquisadores por serem causadores de estresse, modificadores do ambiente e aumentarem a probabilidade do aparecimento de problemas emocionais interferindo no comportamento do indivíduo ${ }^{2,7}$

As variáveis ambientais podem ser consideradas como eventos estressores que podem ser definidos como ocorrências de vida que alteram o ambiente e provocam uma tensão que interfere nas respostas emitidas pelos indivíduos ${ }^{1}$. Tratando-se do período da infância, podem causar impacto sobre a vida cotidiana da criança ou do adolescente devido à ocorrência de mudança na estrutura familiar. Assim, à medida que o ambiente familiar pode afetar aspectos da saúde so- cial e emocional da criança, deve ser considerado um fator importante para o bem-estar da mesma prevenindo uma interferência negativa em seu desenvolvimento normal ${ }^{7-9}$.

Apesar de algumas evidências sobre a influência do ambiente na saúde mental infantil, ainda poucos estudos investigam atualmente a relação entre eventos estressores na família e problemas de saúde mental na infância ${ }^{3,8,10}$. Sendo assim, este estudo tem por objetivo avaliar a relação entre eventos estressores ocorridos na família no último ano e indicativos de problemas de saúde mental em crianças com idade escolar em duas escolas de uma cidade no sul do Brasil.

\section{Metodologia}

Trata-se de um estudo transversal realizado com 1.075 estudantes matriculados em duas escolas públicas de ensino fundamental (uma municipal e outra estadual) do bairro de uma cidade do sul do Brasil. Este faz parte de um estudo maior que teve por objetivo identificar as prevalências de transtornos de desenvolvimento do aprendizado (dislexia e discalculia), transtornos comportamentais e fatores estressores nas famílias e nos professores. A amostra foi considerada de conveniência devido à escolha de escolas próximas à Faculdade de Medicina facilitando o acesso dos pacientes às posteriores intervenções relacionadas ao projeto proposto. As entrevistas foram realizadas nos domicílios, por entrevistadoras treinadas e supervisionadas por dois epidemiologistas.

Para avaliação de fatores emocionais e comportamentais da criança, foi utilizado o questionário de capacidades e dificuldades denominado Strengths and Difficulties Questionnaire (SDQ) nos filhos e nos pais. O SDQ é utilizado para triagem de problemas de saúde mental em crianças dos 4 aos 17 anos. Este instrumento foi aplicado por entrevistadoras aos pais de crianças menores de 11 anos. Acima desta idade, foi aplicado nos pais e nas próprias crianças. O instrumento é composto por 25 itens, que são divididos em cinco subescalas, com cinco itens cada uma, resultando em escores de sintomas emocionais, problemas de conduta, hiperatividade, problemas de relacionamento e comportamento prósocial ${ }^{10}$. Os itens das quatro primeiras escalas geram um escore total de dificuldades. Cada escala tem pontos de cortes diferentes que categorizam em anormal, limítrofe e normal. Para análise, foi agrupado em duas categorias: a) normal; b) anormal (anormal e limítrofe) ${ }^{5}$. 
Os eventos estressores foram avaliados através da Escala de Avaliação de Reajustamento Social de Holmes e Rahe (1967) que mede eventos vitais. Trata-se de uma lista de 26 acontecimentos considerados eventos significativos, como divórcio, nascimento de criança ou morte na família, mudanças no trabalho, entre outros. Esses acontecimentos recebem escores que produzem uma probabilidade de que a criança venha a ficar doente devido ao excesso de estresse. Esta escala foi respondida pela mãe das crianças e adolescentes. A escala não apresenta ponto de corte, quanto maior a pontuação, maior o número de eventos estressores ${ }^{11}$.

A variável "eventos estressores" foi gerada mediante análise de componentes principais. Das 14 variáveis de eventos estressores todas tiveram valor acima de 0,20 na matriz de covariância. Extraiu-se o primeiro componente, com um eigen value de 2,87, que explicou $20,5 \%$ da variância de todas as variáveis. Posteriormente, para fins de análise, este componente foi dividido em tercis, onde o menor representa o grupo com menos eventos estressores.

A análise descritiva dos dados foi feita mediante prevalência. Para a análise bruta da associação entre eventos estressores e cada escala do SDQ, utilizou-se o teste qui-quadrado. Para a análise ajustada, empregou-se regressão de Poisson, com ajuste robusto para variância, expressando os resultados em razão de prevalências (RP) e intervalos de confiança de 95\% (IC95\%). O valor-p usado foi o do teste de Wald para tendência linear. As variáveis colocadas no modelo como possíveis fatores de confusão foram: sexo, idade, reprovação escolar, nível econômico e uso de medicamento contínuo pela mãe. As análises foram realizadas través do programa estatístico Stata, versão 10.0 (Stat Corp, College Station, USA). O nível de significância foi de $5 \%$ para testes bicaudais.

Este estudo contou com o patrocínio da Secretaria Estadual de Saúde do Rio Grande do Sul, através de seu Programa de Prevenção da Violência, e da UNESCO. Todos os responsáveis pelos alunos assinaram um Termo de Consentimento Livre e Esclarecido (TCLE), manifestando sua concordância em participar do estudo, e o projeto foi aprovado pelo Comitê de Ética da Universidade Federal de Pelotas (UFPel).

\section{Resultados}

Das 1,119 crianças matriculadas nas duas escolas, foram realizadas 1,075 entrevistas domiciliares, com uma perda de $4,0 \%$.

Por não existir diferença estatisticamente significativa entre as duas escolas nas características socioeconômicas e de instrução materna, foi considerada, para efeito da análise, a soma dos alunos das escolas. Do total da amostra, 52,7\% (566) são do sexo masculino e 39,6\% (424) tinham entre 12 e 17 anos. A média de idade foi de $10,6$ anos (DP $\pm 2,8)$ e $56,5 \%$ (607) das crianças estavam entre a $1^{\text {a }}$ e $4^{\text {a }}$ séries. Aproximadamente $50 \%$ das crianças já haviam reprovado de série na escola e 60,4\% (644) pertenciam à classe socioeconômica C. Mais de um terço das mães (37\%) relatou usar medicamento de uso contínuo. A prevalência de escore anormal para o SDQ variou de $25 \%$ (escala de relacionamento) a $36 \%$ (escala de conduta) (Tabela 1).

Houve associação entre eventos estressores com cada domínio do SDQ (Tabela 2). Essa associação permaneceu na análise ajustada (Tabela 3). O tercil intermediário de eventos estressores teve 1,34 (IC 95\% 1,08-1,66) vezes mais problemas emocionais quando comparados ao tercil inferior, ao passo que esta chance foi 1,42 (IC 95\% 1,15-1,75) vezes maior se tratando do tercil superior. Quanto aos problemas de conduta, o tercil intermediário apresentou 1,32 (IC 95\% 1,03-1,69) mais chance de ter problemas de conduta e o tercil superior 1,71 (IC 95\% 1,34-2,16), quando comparados ao tercil inferior.

As chances de apresentar problemas de hiperatividade foram 1,42 (IC 95\% 1,10-1,83) vezes maiores no tercil intermediário e 1,37 (IC 95\% 1,06-1,78) no tercil superior, quando comparados ao tercil inferior. Em relação aos problemas de relacionamento, as chances foram de 1,49 (IC $95 \% 1,15-1,93)$ vezes maiores no tercil superior ao serem comparados com o tercil inferior, não apresentando relação com o tercil intermediário (Tabela 3).

\section{Discussão}

O presente estudo avaliou a relação entre eventos estressores ocorridos na família no último ano e indicativos de problemas de saúde mental em crianças com idade escolar em duas escolas de uma cidade no sul do Brasil. Os quatro tipos de problemas investigados pelo instrumento SDQ (emocional, conduta, hiperatividade e relacio- 


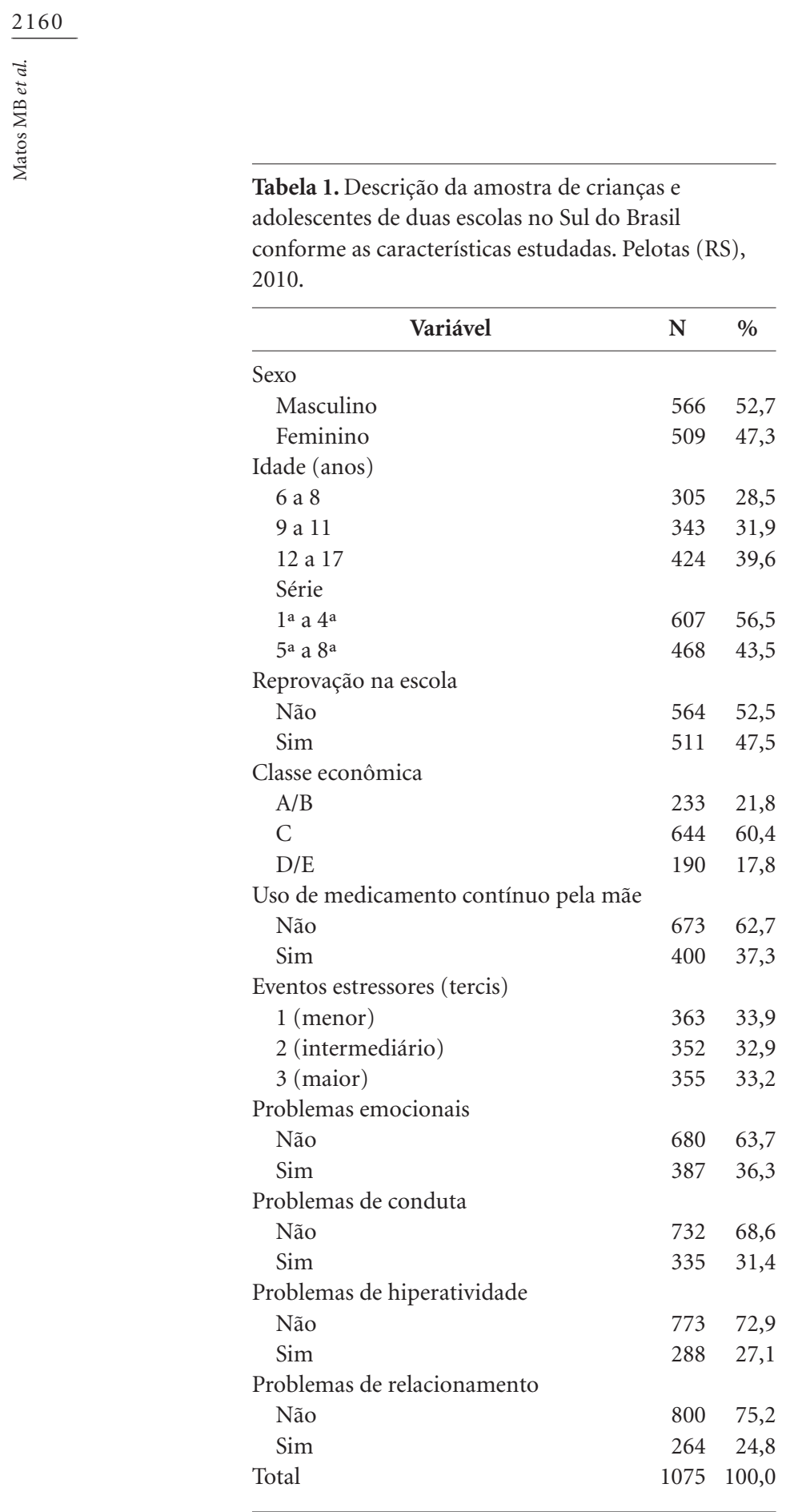

namento com os pares) estiveram associados ao maior relato de eventos estressores no último ano, tanto na análise bruta, como na ajustada (exceto a relação entre o tercil intermediário e os problemas de relacionamento na análise ajustada).

A associação entre os eventos estressores e os problemas de saúde mental em crianças e adolescentes ressalta a interferência causada pelo ambiente. De acordo com a literatura, esta interferência é vista, na maioria das vezes, como prejudicial para o indivíduo e a intensidade desse prejuízo depende do contexto em que vivem e de que maneira a família e a criança/adolescente enfrentam estes eventos ${ }^{12}$. O estresse pode ainda causar sintomatologias especificas, tais como cansaço, confusão mental, prejuízo de memória, apatia, isolamento, depressão, irritabilidade, entre outros ${ }^{13}$, ocasionando baixo rendimento escolar e prejuízos graves que podem aumentar comportamentos de risco, tais como abuso de substâncias e distúrbios alimentares ${ }^{14}$. Desta forma, supõe-se que o impacto destes eventos torna-se maior nos que são mais suscetíveis ou vulneráveis e, consequentemente, podem vir a gerar problemas de saúde mental.

Quanto aos problemas emocionais na infância e adolescência, alguns estudos evidenciam que crianças que sofreram eventos estressantes na vida têm um risco maior de desenvolver problemas emocionais, porém, estas investigações não são atuais ${ }^{15-17}$. Para Harland et al. ${ }^{18}$, existe associação entre as características familiares e o risco de problemas emocionais e de comportamento na criança, sendo o desemprego na família e a separação recente dos pais os fatores mais importantes nesse aumento de risco.

Em relação aos problemas de conduta nas crianças, de acordo com Silva e Hutz ${ }^{19}$ os fatores de risco contextuais (familiares, sociais e experiências de vida negativas ou estressantes) seriam

Tabela 2. Prevalência de problemas de saúde mental, conforme o tercil de eventos estressores $(\mathrm{N}=1075)$. Pelotas, RS, 2010.

\begin{tabular}{lcccc}
\hline Eventos estressores & \multicolumn{3}{c}{ SDQ (escala) } & \\
\cline { 2 - 4 } & $\begin{array}{c}\text { Problemas } \\
\text { Emocionais (\%) }\end{array}$ & $\begin{array}{c}\text { Problemas de } \\
\text { Conduta (\%) }\end{array}$ & $\begin{array}{c}\text { Hiperatividade } \\
(\%)\end{array}$ & $\begin{array}{c}\text { Problemas de } \\
\text { Relacionamento (\%) }\end{array}$ \\
\hline Tercil inferior & 27,9 & 22,4 & 20,6 & 20,1 \\
Tercil intermediário & 37,8 & 30,1 & 29,6 & 22,0 \\
Tercil superior & 43,2 & 41,8 & 31,4 & 32,5 \\
Valor p & $<0,001$ & $<0,001$ & 0,003 & $<0,001$ \\
\hline
\end{tabular}

* Teste do qui-quadrado. 
Tabela 3. Análise ajustada ${ }^{\S}$ entre eventos estressores e os componentes do SDQ $(\mathrm{N}=1075)$. Pelotas, RS, 2010

\begin{tabular}{lcccc}
\hline \multirow{2}{*}{ Eventos estressores } & \multicolumn{3}{c}{ SDQ anormal (escala) } & \\
\cline { 2 - 4 } & $\begin{array}{c}\text { Emocional } \\
\text { RP (IC95\%) }\end{array}$ & $\begin{array}{c}\text { Conduta } \\
\text { RP (IC95\%) }\end{array}$ & $\begin{array}{c}\text { Hiperatividade } \\
\text { RP (IC95\%) }\end{array}$ & $\begin{array}{c}\text { Relacionamento } \\
\text { RP (IC95\%) }\end{array}$ \\
\hline Tercil inferior & 1,00 & 1,00 & 1,00 & 1,00 \\
Tercil intermediário & $1,34(1,08-1,66)$ & $1,32(1,03-1,69)$ & $1,42(1,10-1,83)$ & $1,07(0,80-1,41)$ \\
Tercil superior & $1,42(1,15-1,75)$ & $1,71(1,34-2,16)$ & $1,37(1,06-1,78)$ & $1,49(1,15-1,93)$ \\
Valor $\mathrm{p}^{*}$ & 0,001 & $<0,001$ & 0,02 & 0,003 \\
\hline
\end{tabular}

RP: razão de prevalência; IC95\%: intervalo de confiança de $95 \% .{ }^{\circledR}$ Análise ajustada para sexo, idade, nível econômico, reprovação escolar e uso de medicamento contínuo pela mãe. "Teste de Wald para tendência linear.

um dos fatores preditores para o comportamento delinquente ou transtornos de conduta. Um estudo que investigou os eventos estressores vivenciados por meninas adolescentes que cumpriam medidas socioeducativas, identificou uma ocorrência de alto impacto de pelo menos dois eventos estressores em seus diferentes domínios ${ }^{20}$.

Quanto à hiperatividade na infância e na adolescência, a ocorrência de eventos estressores na família pode causar impacto no rendimento escolar acarretando em dificuldades de concentração, problemas de memória e principalmente comportamento hiperativo ${ }^{21}$. Outro estudo com crianças escolares observou que quanto mais eventos estressores, relacionados principalmente a perdas familiares, mais elas apresentaram dificuldades de adaptação na escola ${ }^{22}$.

De acordo com Ferriolli et al. ${ }^{3}$, a discórdia conjugal, a desvantagem socioeconômica e o alto número de pessoas na família são considerados fatores de risco para problemas de déficit de atenção e, principalmente, hiperatividade. Esta relação torna-se mais consistente no período da infância, já que os problemas de externalização são característicos desta fase do desenvolvimento, ao contrário do período da adolescência, quando os problemas de relacionamento são mais percebidos.

Existem poucos estudos que abordem problemas de relacionamento na infância, tratando-se da adolescência, Dell'Aglio ${ }^{23}$ sugere que as dificuldades no relacionamento com irmãos e colegas se constituem como estressores em si. No estudo de Kristensen et al. ${ }^{24}$, com uma amostra de adolescentes, as discussões com amigos(as) e as brigas com irmãos(ãs) mostraram-se mais prevalentes, da mesma forma que a dificuldade de obedecer às ordens dos pais. Nestes estudos a forma como os eventos estressores são abordados os configuram como um problema de relacionamento, não considerando um desencadeador do outro, assim esta relação deve ser melhor investigada ${ }^{23,24}$.

É importante destacar que este estudo apresenta limitações. Sabe-se que a saúde mental infantil pode ser fortemente influenciada por questões genéticas e estas não foram investigadas. Outra limitação deste estudo é a amostra de conveniência, que englobou duas escolas vizinhas à Faculdade de Medicina, o que limita o poder de generalização e de inferência a partir dos achados. Por último, uma limitação geral de pesquisas transversais diz respeito à questão da causalidade das relações estudadas.

Este é um tema largamente estudado na área biomédica e pouco pesquisado na área da psicologia/psiquiatria. Devido aos prejuízos causados, este tema se torna de extrema relevância em nossa área, necessitando de mais estudos que investiguem a fundo fatores ambientais e sociais considerados de risco para o desenvolvimento da criança e do adolescente.

Através disso, são necessárias novas implementações com modelos de intervenção em serviços comunitários e políticas públicas para reduzir e prevenir estes problemas. De acordo com o Ministério da Saúde ${ }^{25}$, no Brasil, a única proposta para saúde mental das crianças e adolescentes é voltada para os transtornos mentais graves, através da implantação dos Centros de Atenção Psicossocial para a Infância e Adolescência (CAPSi), fato este que nos mostra a importância de novas estratégias de melhoramento e expansão de serviços voltados para esta problemática. 


\section{Colaboradores}

MB Matos participou da avaliação das crianças e adolescentes e da elaboração do artigo; ACN Cruz da coordenação do estudo e revisão do artigo; SC Dumith foi responsável pelas análises estatísticas e revisão do artigo; NC Dias e RBP Carret participaram da avaliação das crianças e adolescentes e da revisão do artigo; e LA Quevedo da coordenação do estudo e orientação do artigo.

\section{Agradecimentos}

Expressamos nossos sinceros agradecimentos ao Dr. Danilo Rolim de Moura (in memorian), criador desta pesquisa, sem a qual a realização deste presente trabalho não teria sido possível. A publicação deste artigo representa, além de uma homenagem, a demonstração de um trabalho desenvolvido com o intuito de priorizar a saúde mental infantil, objetivo este observado em todos os âmbitos de sua carreira.

À Secretaria Estadual de Saúde do Rio Grande do Sul, através do Programa de Prevenção da Violência e da Unesco, pelo financiamento da pesquisa. 


\section{Referências}

1. Sapienza G, Pedromônico MRM. Risco, proteção e resiliência no desenvolvimento da criança e do adolescente. Psicologia em Estudo 2005; 10(2):209-216.

2. Halpern R, Figueiras AC. Environmental influences on child mental health. J Pediatr 2004; 80(Supl. 2):104110.

3. Ferriolli SHT, Marturano EM, Puntel LP. Contexto familiar e problemas de saúde mental infantil no Programa Saúde da Família. Rev Saude Publica 2007; 41(2):251-259.

4. Stewart-Brown S. Research in relation to equity: extending the agenda. Pediatrics 2003; 112(3 Part 2):763-765.

5. Goodman R. Psychometric properties of the Strengths and Difficulties Questionnaire (SDQ). J Am Acad Child Adolesc Psychiatry 2001; 40(11):1337-1345.

6. Santos HV, Pacheco MMDR. Fatores de risco ao desenvolvimento da criança : da visão biomédica à visão psicossocial. Anais do The 4th International Congress on University-Industry Cooperation; novembro 5-7, 2012; Taubate, SP.

7. Rajmil L, Palacio-Vieira JA, Herdman M, López-Aguilà S, Villalonga-Olives E, Valderas JM, Espallargues $\mathrm{M}$, Alonso J. Effect on health-related quality of life of changes in mental health in children and adolescents. Health Qual Life Outcomes 2009; 7:103.

8. Vanaelst B, Huybrechts I, De Bourdeaudhuij I, Bammann K, Hadjigeorgiou C, Eiben G, Konstabel K, Michels N, Molnar D, Moreno LA, Pigeot I, Reisch L, Siani A, Vyncke K, De Henauw S; IDEFICS Consortium. Prevalence of negative life events and chronic adversities in European pre- and primary-school children: results from the IDEFICS study. Arch Public Health 2012; 70(1):26.

9. Waldfogel J, Craigie TA, Brooks-Gunn J. Fragile Families and Child Wellbeing. Future Child 2010; 20(2):87112.

10. Panter-Brick C, Goodman A, Tol W, Eggerman M. Mental health and childhood adversities: a longitudinal study in Kabul, Afghanistan. J Am Acad Child Adolesc Psychiatry 2011; 50(4):349-363.

11. Savoia MG. Escalas de eventos vitais e de estratégias de enfrentamento (coping). Rev Psiq Clin 1999; 26:57-67.

12. Haggerty RJ, Sherrod LR, Gamezy N, Rutter M. Stress, risk and resilience in children and adolescents: process, mechanisms and interventions. J Child Psychol Psychiatry 2000; 37(2):237.

13. Charney DS. Psychobiological mechanisms of resilience and vulnerability: Implications for successful adaptation to extreme stress. Am J Psychiatry 2004; 161(2):195-216.

14. Calais SL, Andrade LMB, Lipp MEN. Diferenças de sexo e escolaridade na manifestação de stress em adultos jovens. Psicologia: Reflexão e Crítica 2003; 16(2):257-263.

15. Hetherington EM, Stanley-Hagen M. The adjustment of children with divorced parents: A risk and resiliency perspective. J Child Psychol Psychiatry 1999; 40(1):129140.
16. Shaw DS, Keenan K, Vondra JI, Delliquadri E, Giovanelli J. Antecedents of preschool children's internalizing problems: A longitudinal study of low-income families. J Am Acad of Child Adolesc Psychiatry 1997; 36(12):1760-1767.

17. Thompson MP, Kaslow NJ, Kingree JB, King M, Bryant Junior M, Rey M. Psychological symptomatology following parental death in a predominantly minority sample of children and adolescents. J Clin Child Psychol 1998; 27(4):434-441.

18. Harland P, Reijneveld SA, Brugman E, Verloove-Vanhorick SP, Verhulst FC. Family factors and life events as risk factors for behavioural and emotional problems in children. Eur Child Adolesc Psychiatry 2002; 11(4):176-184.

19. Silva DFM, Hutz CS. Abuso infantil e comportamento delinqüente na adolescência: prevenção e intervenção. Situações de risco e vulnerabilidade na infância e na adolescência: aspectos teóricos e estratégias de intervenção. São Paulo: Casa do Psicólogo; 2002. p. 151-185.

20. Dell'Aglio DD, Benetti SPC, Deretti L, D'Incao DB, Leon JS. Eventos estressores no desenvolvimento de meninas adolescentes cumprindo medidas sócio-educativas. Paidéia 2005; 15(30):119-129.

21. Lipp MEN. A influência do stress excessivo no desenvolvimento da criança. Mecanismos Neuropsicofisiológicos do Stress: Teoria e aplicação clínica. São Paulo: Casa do Psicólogo; 2003. p. 80-83.

22. Castoldi L, Lopes RCS, Prati LE. O Genograma como Instrumento de Pesquisa do Impacto de Eventos Estressores na Transição Família-Escola. Psicologia: Reflexão e Crítica 2006; 19(2):292-300.

23. Dell'Aglio DD. O processo de coping, institucionalização e eventos de vida em crianças e adolescentes [tese]. Porto Alegre: Universidade Federal do Rio Grande do Sul; 2000.

24. Kristensen CH, Leon JS, D’Incao DB, Dell'Aglio DD. Análise da freqüência e do impacto de eventos estressores em uma amostra de adolescentes. Interação em Psicologia 2004; 8(1):45-55.

25. Brasil. Ministério da Saúde (MS). Caminhos para uma política de saúde mental infanto- juvenil. Brasília: MS; 2005

Artigo apresentado em 26/06/2014

Aprovado em 12/05/2015

Versão final apresentada em 14/05/2015 